\title{
Multijets in Photoproduction and Minijets in DIS at HERA
}

\author{
Albert Knutsson \\ on behalf of the H1 and ZEUS collaborations \\ DESY \\ Notkestr.85, 22603 Hamburg
}

\begin{abstract}
Two measurements based on electron-proton collision data collected at HERA are presented. From the ZEUS collaboration measurements are presented on 3- and 4-jet cross sections in photoproduction. H1 collaboration is for the first time dedicating an analysis to search for multiple interactions in DIS. Both analyses show results which are pointing out the relevance of contributions from the underlying event.
\end{abstract}

\section{Introduction}

Neutral current electron-proton collisions at HERA can be separated into two classes of photon interactions. In the direct photon interaction the photon interacts as a point like particle, while in the resolved photon interaction the photon fluctuates into a partonic system. This leads to a hadron-hadron like scenario, where remnant-remnant interactions may occur in addition to the primary ones. This means that more than one parton from each side may take part in the interaction, resulting in multiple parton interactions (MPI). Since the fraction of resolved events is larger in photoproduction than in DIS, this is where MPI were first observed in inclusive measurements at HERA [1].

Using a total integrated luminosity of $121 \mathrm{pb}^{-1}$ ZEUS measures 3- and 4-jet cross sections in photoproduction. This measurement gives a good tool to study higher order QCD, such as fixed order calculations and QCD models with parton showers, and search for signals of (hard) MPI.

MPI are expected also to take place in direct photon processes where, for example, one of the leading quarks at the photon vertex could interact twice with the proton remnant. In such an event an increase in multiplicity of soft radiations is expected. H1 is searching for (soft) multiple interactions in DIS by measuring the multiplicity of additional soft jets, so called mini jets, in inclusive jet and dijet events.

\section{Event Selection}

The analyses are performed on HERA data, where electrons and protons were collided at an energy of 27.9 respectively $920 \mathrm{GeV}$, giving a center of mass energy of $\sqrt{s}=318 \mathrm{GeV}$. The 3- and 4-jet analysis in addition combines this data with data from run periods where the protons were accelerated to $820 \mathrm{GeV}$.

For both the measurements jets are reconstructed from objects of combined tracks and clusters by the inclusive $k_{t}$-algorithm in the $p_{t}$-recombination scheme. Both measurements are corrected for detector effects, such as limited resolutions and acceptance, binby-bin.

\subsection{3 - and 4-jet final states in Photo- production}

ZEUS is measuring [2] photoproduction events within the kinematical region $Q^{2}<$ $1 \mathrm{GeV}^{2}$ and $0.2<y<0.85$, where $Q^{2}$ and $y$ 
are the virtuality and inelasticity of the photon. Events with at least 3 or 4 jets with transverse momenta $E_{T}>6 \mathrm{GeV}$ and pseudorapidity $\left|\eta_{\text {jet }}^{\text {lab }}\right|<2.4$ are selected. These events are divided into two subsamples with respect to the invariant mass of the $n$-jet system, $25<M_{n j}<50 \mathrm{GeV}$ respectively $M_{n j}>50 \mathrm{GeV}$.

\subsection{Minijet Production in DIS}

$\mathrm{H} 1$ is analysing DIS events within the kinematical range $5<Q^{2}<100 \mathrm{GeV}^{2}, 0.1<y<$ 0.7 and $W>200 \mathrm{GeV}$, where the requirement on the invariant mass of the hadronic final state, $W$, enhance minijet production and events with low $x_{\mathrm{Bj}}$. The results are presented for an inclusive jet sample, containing at least one jet, and a dijet sample in which a subleading jet $(s j)$ is required to be roughly back-to-back to the leading jet $(l j)$ according to $\left|\Delta \phi^{*}\right|=\left|\phi_{l j}^{*}-\phi_{s j}^{*}\right|<140^{\circ}$, where $\phi^{*}$ denotes the azimuthal angle in the hadronic center of mass system. The jets are required to fulfill $E_{T}>5 \mathrm{GeV}$ and $-1.7<\eta_{\text {jet }}^{\text {lab }}<$ 2.79 . In this case the $E_{T}$ requirement is applied in both the laboratory and the hadronic center of mass frame.

The average multiplicity of jets with $E_{T}>$ $3 \mathrm{GeV}$, referred to as minijets, are measured in different azimuthal regions relative to the leading jet. These regions are sketched in Fig. 1. The high and low activity regions are defined event by event and with respect to which region that has the highest and lowest total transverse energy.

\section{QCD Models}

The measurements are compared to theoretical predictions from the Monte Carlo simulations, RAPGAP [3], HERWIG [4] and PYTHIA [5]. These are based on leadingorder matrix elements supplemented with parton shower in the the DGLAP scheme, while the ARIADNE program is based on the Color Dipole Model (CDM) [6].

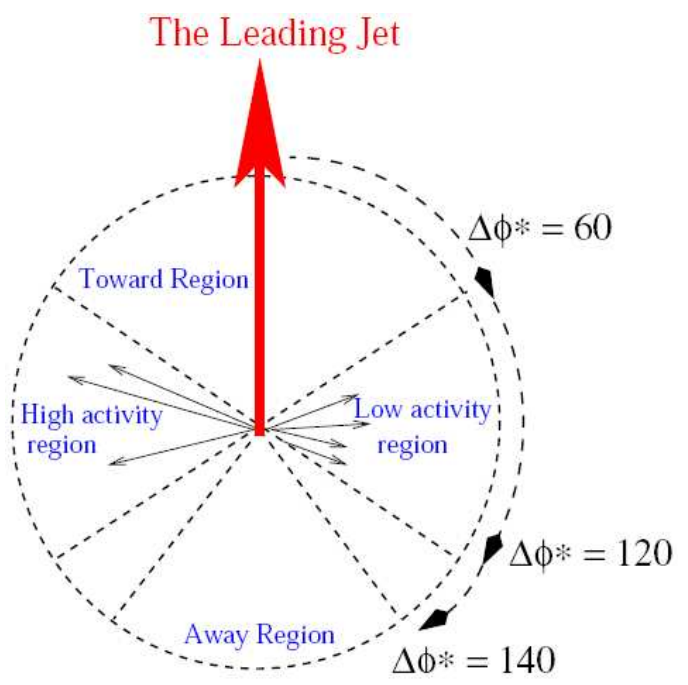

Figure 1: Definition of different azimuthal regions used in the minijet analysis in DIS.

HERWIG and PYTHIA have the possibility to add impact parameter dependent MPI events. In HERWIG this is done by using the add-on package JIMMY [7]. In electronproton scattering the MPI simulations in the programs are so far only fully developed for remnant-remnant interaction. The default values of parameters are used in the predictions presented here.

In addition, the 3 -jet cross sections in photoproduction are compared to a next-toleading order $\mathcal{O}\left(\alpha, \alpha_{s}^{2}\right)$ calculation $[8,9]$.

\section{Results}

\subsection{Multijets in Photoproduction}

In Figs. 2 and 3 the 3 - and 4-jet cross sections as a function of the invariant mass of the jet systems are shown. The statistical errors of the data are represented by the inner error bars, while the outer error bars represent the systematic and the statistical errors added in quadrature. The shaded band following the data points represents the systematic error from the calorimeter energy scale 
uncertainty. The measurement is compared to HERWIG and PYTHIA with and without contributions from MPI, and with the direct and the resolved contribution summed. For HERWIG, also the contribution from direct photon events is shown separately. Each Monte Carlo prediction has been area normalized to the data in the high mass region $\left(M_{n j}>50 \mathrm{GeV}\right)$, as indicated by the scaling factors given in the legend. The result is a reasonable description of the data in the high mass region. However, at low invariant masses the data cross sections are only described by the models if MPI events are included in the simulation. In that case PYTHIA performs slightly better compared to HERWIG. This behaviour is most significant for the 4-jet final state.

The 3 -jet cross section as a function of the invariant mass of the jet system is also compared to an $\mathcal{O}\left(\alpha \alpha_{s}^{2}\right)$ prediction, which has been corrected for hadronization and MPI effects by using HERWIG and PYTHIA. The predictions, corrections and theoretical uncertainties are shown in Fig. 4. The MPI corrections, which are necessary in order for the calculation to describe the data, are increasing with lower invariant mass by up to a factor of 2. After these corrections the data are described by the calculations within the fairly large theoretical uncertainty.

\subsection{Minijet Production in DIS}

In Fig. 5 the average minijet multiplicity as a function of the transverse momentum of the leading jet is presented for the inclusive jet sample. This is done in bins of $Q^{2}$ and for the four different azimuthal regions defined in section 2.2 and in Fig. 1.

The left figure shows comparions to RAPGAP and CDM. The RAPGAP predictions contains the contributions from both direct and resolved events. Neither RAPGAP nor CDM have any MPI implemented, and in the transverse regions where MPI signals are expected to show up, these models predict too

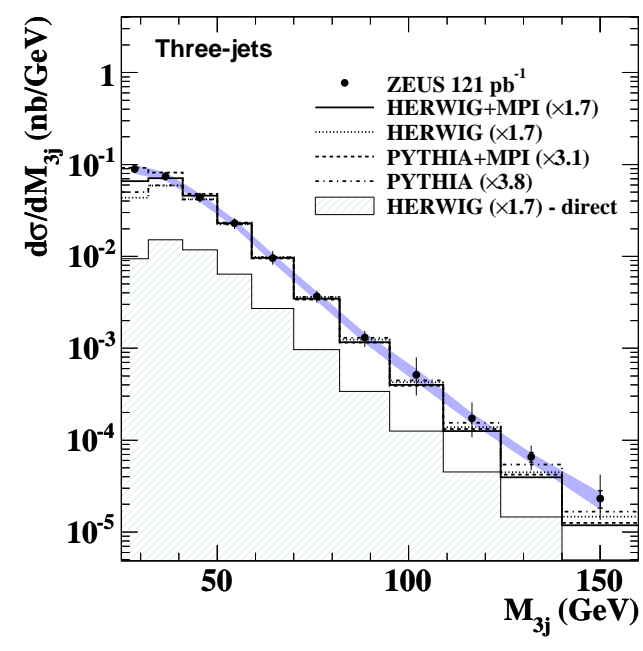

Figure 2: 3 -jet cross sections as a function of the invariant mass system of the 3 -jet system. The QCD models and the errors of the data points are described in the text.

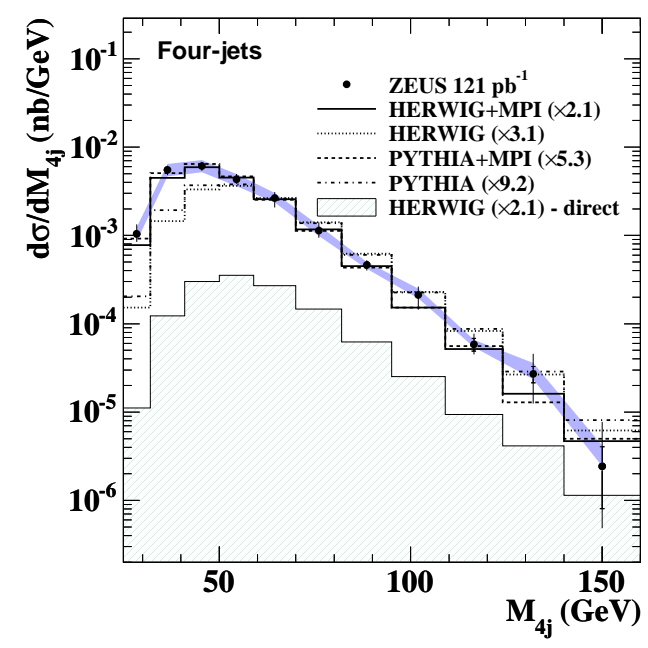

Figure 3: 4-jet cross sections as a function of the invariant mass system of the 4-jet system. The QCD models and the errors of the data points are described in the text. 


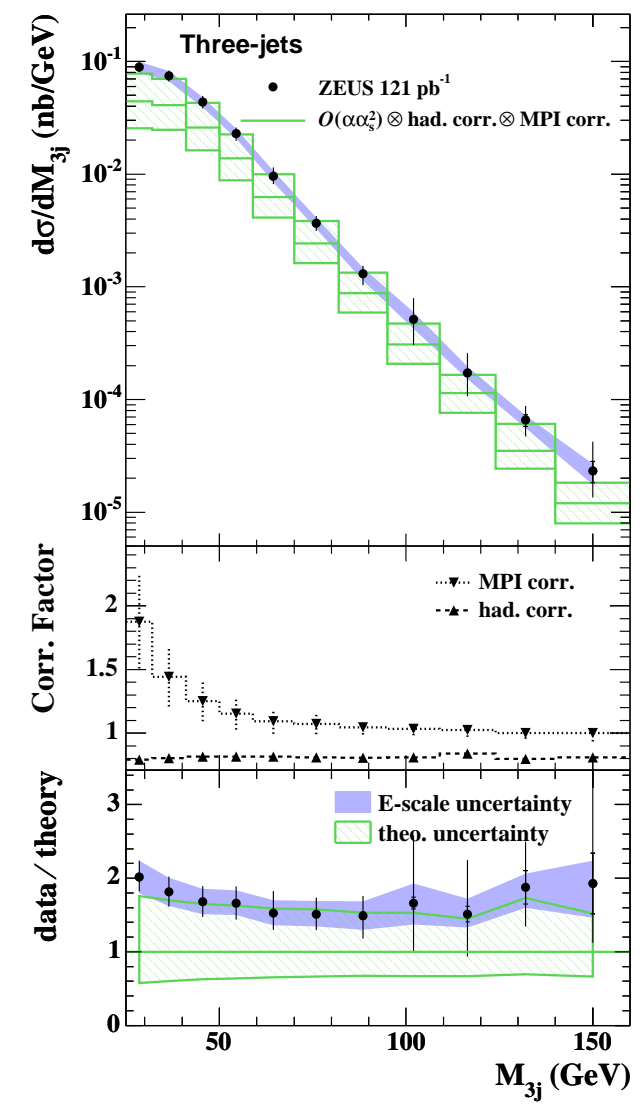

Figure 4: 3-jet cross sections as a function of the invariant mass system of the 3 -jet system. The fixed order calculation, and the errors of the data points are described in the text. low minijet multiplicity. This is most significant at low $Q^{2}$ in the high active region. In the toward and the away region, where the hard physics dominates, the data is however reasonable described.

The right figure shows the same data compared to PYTHA, with and without MPI. In the low $Q^{2}$ bins, where the resolved component is large, the inclusion of MPI events makes a clear improvement of the description of the data in the transverse regions, while at high $Q^{2}$ the MPI make only very small difference. It should be kept in mind that in the MPI model used for $e p$-scattering in PYTHIA, only remnant-remnant interactions are simulated. This means that for the direct contribution MPI are missing in the simulation. Thus the fact that PYTHIA and PYTHA+MPI gives about the same prediction in the highest $Q^{2}$ bin reflects the fact that there is very little contribution from resolved photons in this bin.

\section{Summary}

Results on 3- and 4-jet cross sections in photoproduction and minijet multiplicity in DIS may indicate that MPI has to be considered in order to describe data. In the photoproduction analysis this is most significant for low invariant mass of the jet system and when the event contains more hard jets. It is also observed that the fixed order calculation only describes the photoproduction data after the large corrections for MPI has been applied. In the DIS analysis the MPI contribution is largest for low $Q^{2}$ and shows up in the azimuthal regions transverse to the leading jet.

In addition to the data presented here both analyses comprise several other results. The 3- and 4-jet cross sections in photoproduction has been measured as a function of several additional variables. For the minijet production in DIS results exist also for more inclusive rapidity regions as well as a dijet sample. These results can be found in [1] and [10]. 

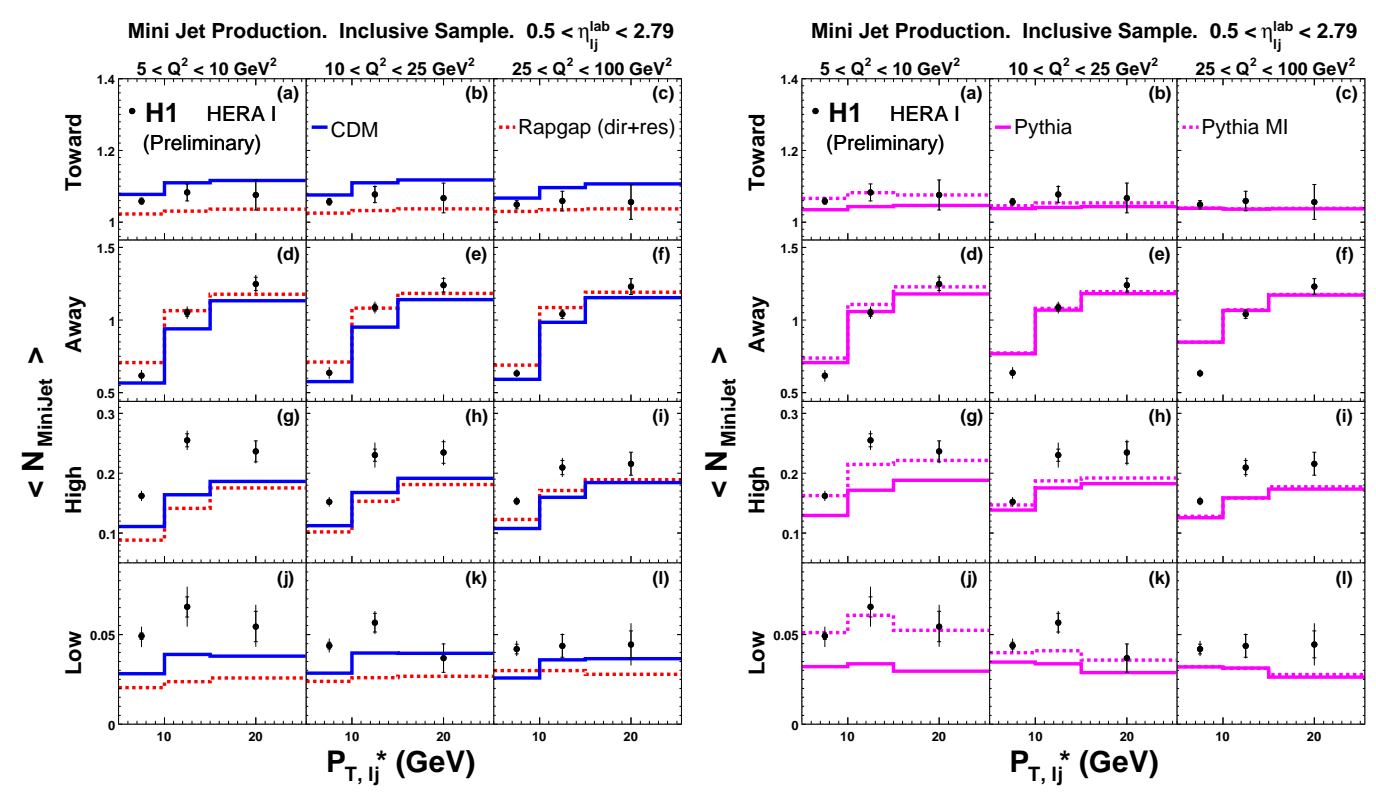

Figure 5: Average minijet multiplicity in bins of $Q^{2}$ in different azimuthal regions with respect the leading jet, as a function of $E_{T}$ of the leading leading jet in the inclusive jet sample. The data is compared to QCD models without MPI (left) and with MPI (right).

\section{References}

[1] S. Aid et al. [H1 Collaboration], Z. Phys. C 70 (1996) 17

[2] S. Chekanov et al. [ZEUS Collaboration], arXiv:0707.3749

[3] H. Jung, Comput. Phys. Commun. 86, 147 (1995).

[4] G. Marchesini, B. R. Webber, G. Abbiendi, I. G. Knowles, M. H. Seymour and L. Stanco, Comput. Phys. Commun. 67, 465 (1992).

[5] T. Sjostrand, P. Eden, C. Friberg, L. Lonnblad, G. Miu, S. Mrenna and E. Norrbin, Comput. Phys. Commun. 135, 238 (2001)

[6] L. Lonnblad, Comput. Phys. Commun. 71, 15 (1992).

[7] J.M. Buttherworth and M.H.Seymour, JIMMY4: Multiparton Interactions in HERWIG for the LHC (unpublished), 2005, available on http://hepforge.cedar.ac.uk/jimmy/

[8] M. Klasen, T. Kleinwort and G. Kramer, Eur. Phys. J. direct C 1, 1 (1998)

[9] M. Klasen, Eur. Phys. J. C 7, 225 (1999)

[10] Slides:

http://indico.cern.ch/materialDisplay.py?

Photon 2007

contribId $=32 \&$ session $I d=14$

\&material Id=slides\&conf $I d=3841$ 